\title{
Application of conventional ultrasound coupled with virtual touch tissue imaging and quantification in the assessment of muscle strength
}

\author{
Weichen Zhou ${ }^{1} \wedge$, Xiaojuan $\mathrm{Ma}^{1}$, Lin Pan $^{2}$, Yunhan Wang ${ }^{1}$, Chengting Zhou ${ }^{1}$ \\ ${ }^{1}$ Department of Ultrasound, Chengdu First People's Hospital, Chengdu, China; ${ }^{2}$ Department of Rheumatology and Immunology, Chengdu First \\ People's Hospital, Chengdu, China \\ Contributions: (I) Conception and design: C Zhou; (II) Administrative support: X Ma; (III) Provision of study materials or patients: L Pan, Y Wang; (IV) \\ Collection and assembly of data: W Zhou; (V) Data analysis and interpretation: W Zhou; (VI) Manuscript writing: All authors; (VII) Final approval \\ of manuscript: All authors. \\ Correspondence to: Chengting Zhou. Department of Ultrasound, Chengdu First People's Hospital, 18 N. Wanxiang Rd., Gaoxin District, Chengdu, \\ China. Email: 358323121@qq.com.
}

Background: Grip strength is the gold standard for the assessment of muscle strength. The current
methods of measuring grip strength can only reflect the overall function of the muscle group, not an actual
muscle lesion or the cause of the lesion. Virtual touch tissue imaging and quantification (VTIQ) technology
can provide a representation of muscle properties and quantify alterations in muscle mechanics through
measurements of the mean shear wave velocities of the muscle group at a designated location. Methods: A total of 70 healthy middle-aged females were included. The maximum grip strength and thickness of the forearm and hand muscle under relaxed conditions were measured, and the sum of the muscle thicknesses was calculated. VTIQ was used to measure the shear wave velocity $\left(V_{s}\right)$ of the flexor pollicis longus, flexor digitorum profundus and flexor digitorum superficialis. Intra-group correlation coefficient (ICC) was used to evaluate the test-retest reliability of the grip strength and $V_{s}$ values, and the correlation of the thickness and $V_{s}$ of each forearm and hand muscle with grip strength was calculated.

Results: (I) The hand-held dynamometer and the VTIQ technology have good test-retest reliability in muscle evaluation (all $\mathrm{P}<0.001$ ). (II) The muscle thickness of the lumbricals did not exhibit a significant correlation with grip strength $(\mathrm{P}>0.05)$. The forearm radial MT (muscle thickness), forearm ulna MT, summation of four MTs, and interosseous MT showed positive correlations with grip strength ( $\mathrm{r}=0.445,0.824$, 0.722 , and 0.359 , all $\mathrm{P}<0.05)$. The $\mathrm{V}_{\mathrm{s}}$ values of the flexor pollicis longus, flexor digitorum superficialis, and flexor digitorum profundus showed negative correlations with grip strength ( $\mathrm{r}=-0.962,-0.919$, and -0.456 , all $\mathrm{P}<0.01)$. Stepwise linear regression revealed that forearm radial, ulnar muscle thickness, and flexor digitorum superficialis $V_{s}$ can be utilized concurrently to predict $90.7 \%$ of the variability of grip strength $\left(\mathrm{R}^{2}=0.907\right)$.

Conclusions: The structure and stiffness values of skeletal muscles obtained through conventional ultrasound coupled with VTIQ showed good correlations with the variation in grip strength and can evaluate the changes of muscle strength.

Keywords: Grip strength; ultrasound; elastography; muscle strength

Submitted Aug 03, 2020. Accepted for publication Sep 15, 2020.

doi: 10.21037/apm-20-1715

View this article at: http://dx.doi.org/10.21037/apm-20-1715

\footnotetext{
^ ORCID: 0000-0003-0689-2970.
} 


\section{Introduction}

The skeletal muscle serves as the main actuator of body movement, and its normal structure and mechanical properties are required for basic body movement. Reduction in the mass and strength of skeletal muscle increases the risk of bone fracture and disability; the onset of chronic diseases, including diabetes mellitus, cardiovascular diseases, and cancer; and premature death of the elderly (1). Grip strength is a widely recognized indicator of overall muscle strength and health and an independent predictor of all-cause mortality and the prognosis of cardiovascular and chronic kidney diseases in the community $(2,3)$. Extensively used clinically, grip strength measurement is the only evaluation method recommended by the Asian Working Group for Sarcopenia: 2019 Consensus Update on Sarcopenia Diagnosis and Treatment for measurement of muscle strength (4).

Grip strength is measured mainly with hand dynamometers. As the most extensively used dynamometer, the Jamar dynamometer is currently considered the gold standard for grip strength evaluation. However, the measurement stability of grip strength is low because the results are prone to subjective influences from the tester and the subject (5). Moreover, grip strength can only indirectly reflect the overall condition of the relevant muscle group and is limited by the fact that the effects of specific muscle lesions on grip strength cannot be pinpointed based on the reduction in grip strength.

As a real-time, noninvasive, simple, and high-resolution technology, musculoskeletal ultrasound can not only clearly depict the minute morphological structures of the muscle but also reflect the alterations in muscle structure and function through measurements of muscle thickness, cross-sectional area, fiber length, and pennation angle. In the 1990s, ultrasonic photography was used to evaluate the structural and functional status of muscle (6). Another study found that MT could well reflect the cross-sectional area of muscle (7). Additionally, with the application of elastography in the evaluation of skeletal muscle stiffness, many studies have proposed that muscle stiffness varies with age, exercise status and muscle lesions. A previous study has reported that shear modulus values of the biceps brachii increased with advancing age; however, shear modulus values for females tended to be higher than those for males (8). The shear wave speed and echo intensity of the paretic side were greater than those of the non-paretic side in stroke patients (9).
Additionally, as a form of elastography, quantification (VTIQ) technology is a shear wave elastic imaging technique that is able to evaluate the image quality. It creates a source of mechanical wave inside the tissue by acoustic radiation force impulse. The wave transmits via the vertical direction of the ultrasound wave while generating the shear wave horizontally in the tissue. The trivial change in the tissue caused by the pressure of the mechanical wave will be captured by the device and produce multicolored elastic images. Meanwhile, the speed of shear wave will be calculated by the time difference between two neighboring shear wave peaks and wavelength. It can accurate visualization of muscle stiffness via measurement of the mean shear wave velocity (Vs) of a selected tissue area and enable the evaluation of the mechanical properties of skeletal muscles (10-13).

VTIQ was used to evaluate the stiffness of normal human tissues in previous studies. In this study, the thickness and stiffness of forearm muscles were quantified through conventional ultrasound coupled with VTIQ, and the practicability of this technology in the measurement of the mechanical properties of skeletal muscles was explored. It is the first time to combine the stiffness of tissue with mechanical properties. We present the following article in accordance with the MDAR reporting checklist (available at http://dx.doi.org/10.21037/apm-20-1715).

\section{Methods}

\section{Clinical information}

A total of 70 healthy females aged 40-60 years (average age 49.44 \pm 9.22) were selected from our health examination Center between 2018 and 2019. All subjects were righthanded, and none had any history of chronic diseases (coronary artery disease, valvular heart disease, joint diseases, or neuromuscular diseases), hyperthyroidism, hypothyroidism, recent trauma, or intake of drugs that affect the neuromuscular system. Four subjects with history of hand trauma were excluded. The subject had three practice trials before the test to become familiarized with the proper way to apply force. Poor consistency in practice trials (10 patients) were not included in the study to avoid influencing the results, so 56 were included in the study. All procedures performed in this study involving human participants were in accordance with the Declaration of Helsinki (as revised in 2013). This study followed the International Ethical Guidelines for Biomedical Research 


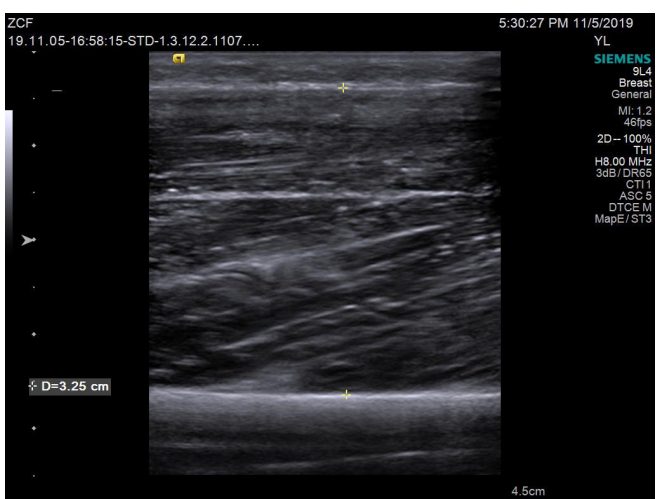

Figure 1 The 2D image of the forearm ulnar muscles of a 52-yearold healthy female. The muscle fibers were continuous, and the echo intensity was homogeneous. The thickness of ulnar muscles was $3.25 \mathrm{~cm}$.

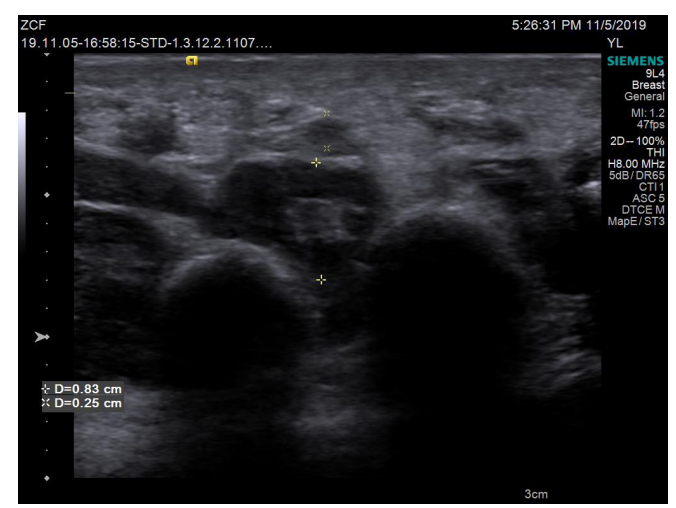

Figure 2 The thickness of the lumbricals in the palm of a 52-yearold healthy female was $0.25 \mathrm{~cm}$, and that of the interosseous muscles was $0.83 \mathrm{~cm}$.

Involving Human Subjects published by the Council for International Organizations of Medical Sciences and was approved by the Ethics Committee of Chengdu First People's Hospital, China (approval number: No. 12 of 2018). Informed consent was obtained from every subject before participation.

\section{Measurement methods}

\section{Grip strength test}

Grip strength was measured with Jamar hand-held dynamometers. The posture for grip strength testing was defined in the Physical Fitness Report: The subject assumed a standing posture, with arms at the side and palms toward the body. After the breathing and heart rates were stabilized, the subject held the dynamometer in the right hand and squeezed the handle of the dynamometer with as much force as possible. The maximum grip strength value was recorded. After 24 hours, the grip strength was measured and recorded again.

\section{Measurement of muscle parameters}

The upper part of the right forearm $(30 \%$ of the way from the radial head to the radial styloid process) and the middle-upper part of the palm (55\% of the way from the wrist flexion crease to the palmar digital crease) of the subject were marked. An ACUSON Oxana 2000 color Doppler ultrasound diagnostic system (SIEMENS) with 9L4 linear array transducers was used in musculoskeletal mode. The subject was in the supine position, with the right palm supinated, the fingers extended and relaxed, and the right forearm exposed. The transducer was placed gently on the marked sites on the right forearm and palm, and longitudinal and transverse scans were conducted. After clear images of the muscle fiber structure were obtained, the vertical distances between the subcutaneous adipose tissuemuscle interface and the muscle-bone interface anterior to both the radius and ulna were measured on the longitudinal view. The distances between the superficial fascia and deep fascia of the dorsal interosseous muscles and lumbricals were measured on the transverse view at the marked position between the middle finger and the ring finger (Figures 1,2). The sum of the thicknesses of the radial, ulnar, interosseous, and lumbrical muscles was calculated. The transducer was placed on the marked site on the forearm with minimal pressure, and the subject was told to bend the interphalangeal joint of the thumb and the distal and proximal interphalangeal joints of Figures 2-5. The radial flexor pollicis longus, ulnar flexor digitorum profundus, and flexor digitorum superficialis were identified, the transducer was aligned with the muscle fiber, and VTIQ imaging mode was applied. The diameter of the field of interest was set at $10 \mathrm{~mm}$. When the sampling area turned uniformly green in QC mode (Figure 3), velocity mode was started, the shear wave velocity of the proximal, middle, distal superficial and deep muscles were measured on the long axis section with a square measuring sampling frame of $1 \mathrm{~mm} \times 1 \mathrm{~mm}$. The average value was obtained by measuring 6 times. The whole process of examination and measurement was completed by the same trained physician with the same technique (Figure 4). The mean of six replicates was calculated for each measurement, and the 


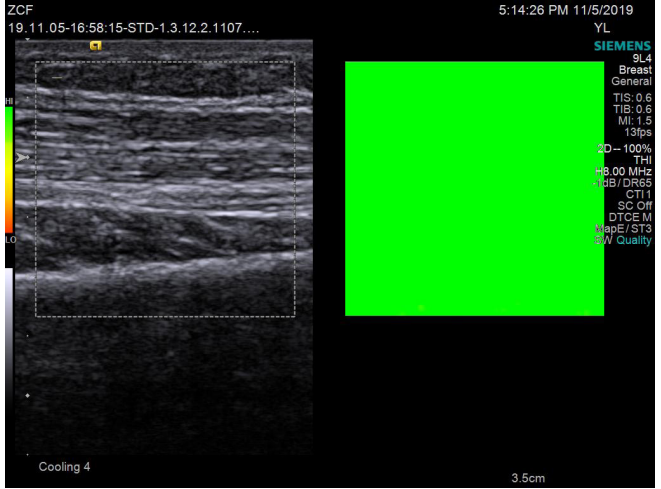

Figure 3 The image of the flexor pollicis longus of a 52-yearold healthy female under VTIQ quality control mode showed a uniform green distribution.

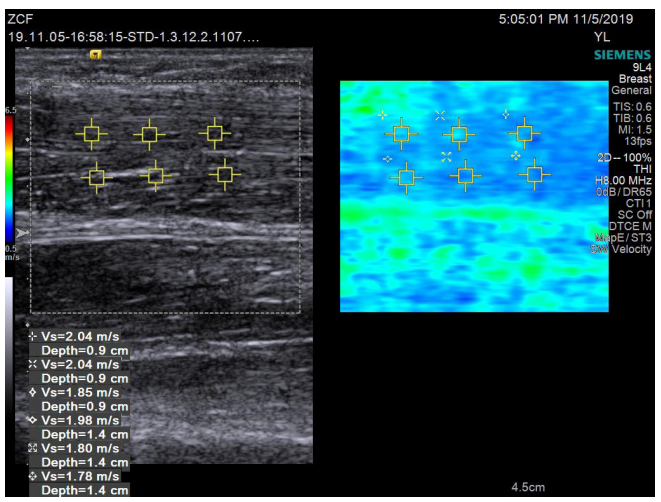

Figure 4 The VTIQ image of the $V_{s}$ of the flexor digitorum superficialis of a 52-year-old healthy female. The mean $V_{s}$ was $1.61 \mathrm{~m} / \mathrm{s}$.

shear wave velocities were measured and recorded in the same fashion 24 hours later.

\section{Statistical analysis}

Measurement data are presented as $(\bar{x} \pm S)$. SPSS 23.0 software was used for statistical analysis. The intraclass correlation coefficient (ICC) was used to evaluate the confidence levels of the measurements of the grip strength and $\mathrm{V}_{\mathrm{s}}$ of forearm muscles 24 hours apart. ICC $>0.75$ indicated high confidence, ICC between 0.5 and 0.75 indicated moderate confidence, and ICC $<0.5$ indicated poor confidence. Pearson's correlation coefficient was calculated to measure the correlations between grip strength and the measurements of muscle thickness and muscle $V_{s}$, with the significance level $\alpha=0.05$. Multiple linear regression was used to identify the measurements that best predicted grip strength.

\section{Results}

\section{The test-retest reliability of the hand-held dynamometer} and VTIQ technology for muscle measurements

The hand-held dynamometer and VTIQ technology exhibited good test-retest reliability in the evaluation of muscles. The ICC was 0.955 for the test-retest reliability of grip strength, 0.866 for the $V_{s}$ of the flexor pollicis longus, 0.930 for the $V_{s}$ of the flexor digitorum superficialis, and 0.920 for the $\mathrm{V}_{\mathrm{s}}$ of the flexor digitorum profundus $(\mathrm{P}<0.01)$ (Table 1).

\section{The measurement data of the forearm muscles of healthy middle-aged females (Tables 2,3)}

The average age of healthy females was $49.44 \pm 9.22$ years. Grip strength was $27.19 \pm 4.07 \mathrm{~kg}$. The thicknesses of the ulnar, radial, lumbrical and interosseous muscles were $2.92 \pm 0.40$, $1.46 \pm 0.13,0.37 \pm 0.06,0.77 \pm 0.14 \mathrm{~cm}$, respectively, and the sum of the thicknesses of four muscles was $5.53 \pm 0.52 \mathrm{~cm}$. The Vs of the flexor pollicis longus, flexor digitorum superficialis, and flexor digitorum profundus were $2.77 \pm 0.38,2.32 \pm 0.27,2.18 \pm 0.25 \mathrm{~m} / \mathrm{s}$, respectively.

\section{Correlation analysis on muscle thickness, $V_{s}$, and grip strength}

The thickness of the lumbricals showed no correlation with grip strength $(r=0.127, \mathrm{P}>0.05)$. The thickness of radial muscles and interosseous muscles showed positive correlations with grip strength $(r=0.445$ and $r=0.359$, respectively, both $\mathrm{P}<0.05)$. The thickness of the ulnar muscles and the combined thickness of the extrinsic and intrinsic muscles showed significant correlations with grip strength $(\mathrm{r}=0.824$ and $\mathrm{r}=0.722$, respectively, both $\mathrm{P}<0.01)$ (Figure 5). The $\mathrm{V}_{\mathrm{s}}$ of the flexor pollicis longus, flexor digitorum superficialis, and flexor digitorum profundus showed negative correlations with grip strength $(\mathrm{r}=-0.962$, $\mathrm{r}=-0.919$, and $\mathrm{r}=-0.456$, respectively, all $\mathrm{P}<0.01)$. The $\mathrm{V}_{\mathrm{s}}$ of the flexor pollicis longus and flexor digitorum superficialis exhibited significant correlations with each other (Figure 6). Stepwise linear regression analysis revealed that concurrent use of radial muscle thickness, ulnar muscle thickness, 
Table 1 The test-retest reliability of hand-held dynamometer and VTIQ technology on muscle measurements

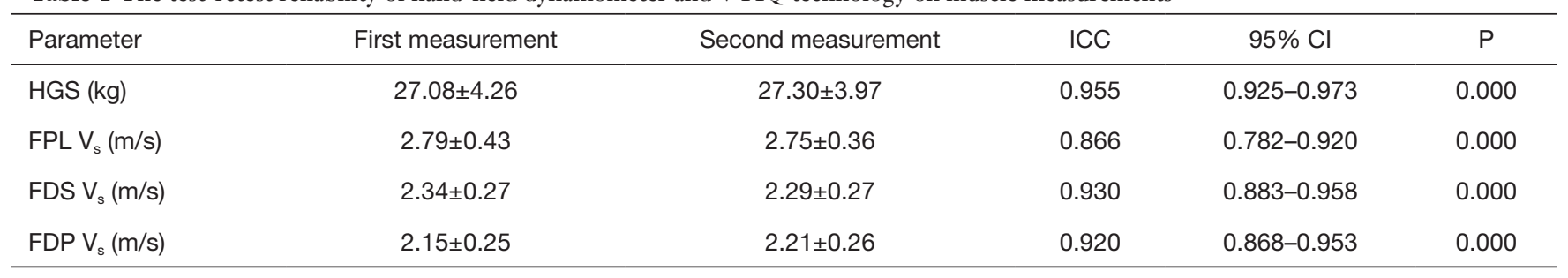

HGS, handgrip strength; ICC, intraclass correlation coefficient; $V_{s}$, shear wave velocity; FPL, flexor pollicis longus; FDS, flexor digitorum superficialis; FDP, flexor digitorum profundus.

Table 2 Muscle thicknesses in healthy middle-aged females

\begin{tabular}{lc}
\hline Parameter & $\bar{x} \pm s$ \\
\hline Forearm ulnar MT (cm) & $2.92 \pm 0.40$ \\
Forearm radial MT (cm) & $1.46 \pm 0.13$ \\
Lumbrical MT (cm) & $0.37 \pm 0.06$ \\
Interosseous MT (cm) & $0.77 \pm 0.14$ \\
Sum MT (cm) & $5.53 \pm 0.52$ \\
\hline
\end{tabular}

MT, muscle thickness.

Table 3 Shear wave velocity $\left(V_{\mathrm{s}}\right)$ of forearm muscles in healthy middle-aged females

\begin{tabular}{|c|c|}
\hline Parameter & $\bar{x} \pm s$ \\
\hline Age (years) & $49.4 \pm 9.22$ \\
\hline HGS (kg) & $27.19 \pm 4.07$ \\
\hline $\mathrm{FPL} \mathrm{V}_{\mathrm{s}}(\mathrm{m} / \mathrm{s})$ & $2.77 \pm 0.38$ \\
\hline FDS $V_{s}(m / s)$ & $2.32 \pm 0.27$ \\
\hline FDP V $(\mathrm{m} / \mathrm{s})$ & $2.18 \pm 0.25$ \\
\hline
\end{tabular}

HGS, handgrip strength; FPL, flexor pollicis longus; FDS, flexor digitorum superficialis; FDP, flexor digitorum profundus.

and the $\mathrm{V}_{\mathrm{s}}$ of the flexor digitorum superficialis predicted $90.7 \%$ of the changes in grip strength $\left(\mathrm{R}^{2}=0.907\right)$, with the regression equation $\mathrm{Y}=49.29-5.02 \mathrm{X}_{2}-5.70 \mathrm{X}_{5}+1.07 \mathrm{X}_{1}$.

\section{Discussion}

After the skeletal muscle fiber receives an electrical stimulus from a nerve, the muscle tone increases (change in mechanical property), and the muscle contracts (mechanical reaction) to complete the musculoskeletal movement. An intact muscle structure and muscle tone are the basic

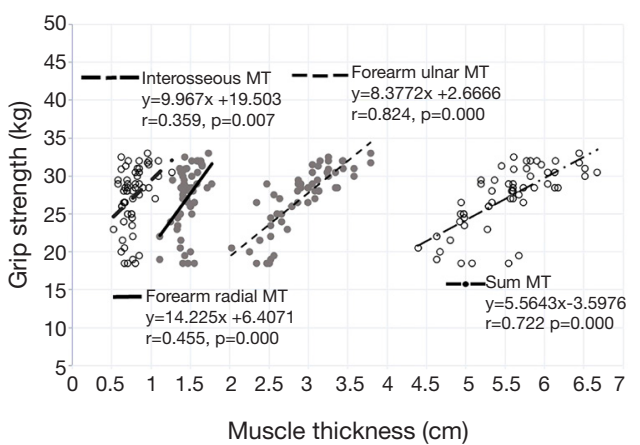

Figure 5 Correlations between grip strength and the thicknesses of forearm and palm muscles.

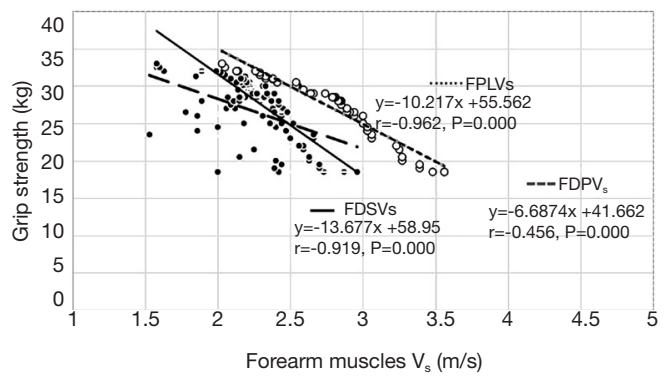

Figure 6 Correlations between shear wave velocities of forearm muscles and grip strength.

requirements to ensure the body can achieve effective movements. Grip strength is a direct reflection of the functions of the hand muscles. The gripping movement is achieved by concerted actions of forearm flexors (extrinsic muscles) and muscles located within the hand (intrinsic muscles) (12-14). The forearm flexors include the flexor digitorum superficialis, flexor digitorum profundus, and flexor pollicis longus. The intrinsic hand muscles include the thenar muscles, hypothenar muscles, interosseous muscles, and lumbrical muscles. Precise evaluation of the 
structure and function of hand muscles is required for the utilization of grip strength as an indicator of the functional status of skeletal muscles and can also reveal the direct causes of reductions in skeletal muscle strength clinically, making it a reliable reference for targeted therapies.

This study used conventional ultrasound to precisely localize the distribution of skeletal muscles, visualize the shape and structural characteristics of the muscle fiber, and quickly and accurately measure muscle thickness. The thicknesses of forearm and palm muscles were found to positively correlate with grip strength, and the thicknesses of ulnar muscles and the combined thickness of extrinsic and intrinsic muscles showed strong correlations with grip strength. The thicker the ulnar muscles, the stronger the grip strength. These findings are consistent with the results reported by Abe et al., which showed that grip strength was positively correlated with ulnar muscle sickness, regardless of age or sex $(14,15)$.

While conventional ultrasound can accurately evaluate changes in muscle morphology and structure, during the early stages of aging and chronic diseases (such as diabetes mellitus, rheumatic diseases, and organ failure), before any observable change in muscle morphology $(16,17)$, the decrease in hemoglobin level, the release of inflammatory cytokines, and changes in hormone levels result in muscle fiber hyperplasia, adipose infiltration, and elevated collagen content (18-20), which lead to elevated muscle stiffness. The increase in muscle stiffness results in decreases in the number of contracting muscle fibers and the extent of their contraction. The reduction in muscle strength is reflected in the reduction in measured grip strength. Meanwhile, the reduction in skeletal muscle function further affects the prognosis of diseases.

During VTIQ, acoustic pulses are emitted into the selected organ or tissue region, where they induce localized deformation and displacement. This tissue can be quantitatively evaluated by measuring the velocities of the waves spreading perpendicular to the excitation beam (shear wave). Thus, the changes in skeletal muscle stiffness can be detected before the muscle morphology changes. Brandenburg et al. (21) measured the stiffness of the lateral head of the gastrocnemius of both legs in normally developing children during passive movement and observed high feasibility and reliability between testers and subjects, indicating that elastography is reliable for the evaluation of muscle stiffness. Du et al. (22) found that the Young's modulus of the biceps brachii of patients with marked symptoms of Parkinson's disease, as well as those of asymptomatic Parkinson patients, were significantly higher than those of healthy controls, and the differences between these three groups were statistically significant (all $\mathrm{P}<0.05$ ). These findings suggest that shear wave elastography can reflect muscle function and provide useful information for the early diagnosis of diseases.

This study found that grip strength decreased when the stiffness of forearm muscles increased. The $V_{s}$ values of the flexor pollicis longus and flexor digitorum superficialis exhibited the strongest correlation with grip strength $(r=-0.962$ and $r=-0.919$, respectively), indicating that muscle stiffness can be used as a quantitative representation of changes in grip strength to a certain degree, reflecting overall changes in muscle strength, and in the early detection of the alterations in muscle morphology and tissue structure that result in the decreased grip strength.

VTIQ technology has the quality mode which can improve the accuracy of measurement, and overcome the shortcomings of traditional pressure elastography, such as relying on the experience of the operator and unable to quantitatively evaluate the elasticity of tissues, it can reflect the stiffness of muscle tissue quantitatively and noninvasively and allow clinicians to assess muscle stiffness via quick measurements of the $\mathrm{V}_{\mathrm{s}}$ of individual muscles. Combined with conventional Grip strength test, VTIQ can precisely localize the muscle lesion and the corresponding functional changes within a muscle group and uncover the changes in skeletal muscle mechanics that lead to reduced grip strength. Therefore, VTIQ may provide an effective diagnostic method of early-stage skeletal muscle functional deterioration caused by aging and chronic diseases, as well as provide a foundation for early clinical intervention through lifestyle changes, the search for potentially reversible causal factors, and the establishment of appropriate personalized treatment plans. However, the skeletal muscle is an anisotropic organ, the orientation of the fibres in the skeletal muscle may probably influence to monitoring precision of VTIQ. No manual pressure was to be applied to the transducer during the examination, so manual applied pressure may affect the elastographic measurement. To determine whether the different orientation of the fibres operator-dependency, or another reason affects accuracy of shear wave velocity, we suggest that further studies are conducted. Furthermore, because the sample size in this study is small, further research is necessary to confirm the practicality of VTIQ in the assessment of muscle strength. 


\section{Acknowledgments}

Funding: Scientific Research Project of Sichuan Health and Family Planning Commission (18PJ141).

\section{Footnote}

Reporting Checklist: The authors have completed the MDAR reporting checklist. Available at http://dx.doi.org/10.21037/ apm-20-1715

Data Sharing Statement: Available at http://dx.doi. org/10.21037/apm-20-1715

Conflicts of Interest: All authors have completed the ICMJE uniform disclosure form (available at http://dx.doi. org/10.21037/apm-20-1715). The authors have no conflicts of interest to declare.

Ethical Statement: The authors are accountable for all aspects of the work in ensuring that questions related to the accuracy or integrity of any part of the work are appropriately investigated and resolved. All procedures performed in this study involving human participants were in accordance with the Declaration of Helsinki (as revised in 2013). This study followed the International Ethical Guidelines for Biomedical Research Involving Human Subjects published by the Council for International Organizations of Medical Sciences and was approved by the Ethics Committee of Chengdu First People's Hospital, China (approval number: No. 12 of 2018). Informed consent was obtained from every subject before participation.

Open Access Statement: This is an Open Access article distributed in accordance with the Creative Commons Attribution-NonCommercial-NoDerivs 4.0 International License (CC BY-NC-ND 4.0), which permits the noncommercial replication and distribution of the article with the strict proviso that no changes or edits are made and the original work is properly cited (including links to both the formal publication through the relevant DOI and the license). See: https://creativecommons.org/licenses/by-nc-nd/4.0/.

\section{References}

1. Chuang SY, Hsu YY, Chen RC, et al. Abdominal Obesity and Low Skeletal Muscle Mass Jointly Predict Total Mortality and Cardiovascular Mortality in an Elderly Asian Population. J Gerontol A Biol Sci Med Sci 2016;71:1049-55.

2. Wu Y, Wang W, Liu T, et al. Association of Grip Strength With Risk of All-Cause Mortality, Cardiovascular Diseases, and Cancer in Community-Dwelling Populations: A Metaanalysis of Prospective Cohort Studies. J Am Med Dir Assoc 2017;18:551.e17-551.e35.

3. Park S, Cho J, Kim D, et al. Handgrip strength, depression, and all-cause mortality in Korean older adults. BMC Geriatr 2019;19:127.

4. Chen LK, Woo J, Assantachai P, et al. Asian Working Group for Sarcopenia: 2019 Consensus Update on Sarcopenia Diagnosis and Treatment. J Am Med Dir Assoc 2020;21:300-7.e2.

5. Roberts HC, Denison HJ, Martin HJ, et al. A review of the measurement of grip strength in clinical and epidemiological studies: towards a standardised approach. Age Ageing 2011;40:423-9.

6. Ikai M, Fukunaga T. Calculation of muscle strength per unit cross-sectional area of human muscle by means of ultrasonic measurement. Int $Z$ Angew Physiol 1968;26:26-32.

7. Abe T, Loenneke JP, Thiebaud RS. Ultrasound assessment of hamstring muscle size using posterior thigh muscle thickness. Clin Physiol Funct Imaging 2016;36:206-10.

8. Eby SF, Cloud BA, Brandenburg JE, et al. Shear wave elastography of passive skeletal muscle stiffness: influences of sex and age throughout adulthood. Clin Biomech (Bristol, Avon) 2015;30:22-7.

9. Lee SS, Spear S, Rymer WZ. Quantifying changes in material properties of stroke-impaired muscle. Clin Biomech (Bristol, Avon) 2015;30:269-75.

10. Lee SS, Gaebler-Spira D, Zhang LQ, et al. Use of shear wave ultrasound elastography to quantify muscle properties in cerebral palsy. Clin Biomech (Bristol, Avon) 2016;31:20-8.

11. Hug F, Tucker K, Gennisson JL, et al. Elastography for Muscle Biomechanics: Toward the Estimation of Individual Muscle Force. Exerc Sport Sci Rev 2015;43:125-33.

12. Eby SF, Song P, Chen S, et al. Validation of shear wave elastography in skeletal muscle. J Biomech 2013;46:2381-7.

13. Brandenburg JE, Eby SF, Song P, et al. Ultrasound elastography: the new frontier in direct measurement of muscle stiffness. Arch Phys Med Rehabil 2014;95:2207-19.

14. Kim T, Huh S, Kim SY, et al. ICU rehabilitation is associated with reduced long-term mortality from sepsis in patients with low skeletal muscle mass: a case control study. Ann Transl Med 2019;7:430.

15. Abe T, Thiebaud RS, Loenneke JP, et al. Association 
between forearm muscle thickness and age-related loss of skeletal muscle mass, handgrip and knee extension strength and walking performance in old men and women: a pilot study. Ultrasound Med Biol 2014;40:2069-75.

16. Akagi R, Yamashita Y, Ueyasu Y. Age-Related Differences in Muscle Shear Moduli in the Lower Extremity. Ultrasound Med Biol 2015;41:2906-12.

17. Eby SF, Cloud BA, Brandenburg JE, et al. Shear wave elastography of passive skeletal muscle stiffness: influences of sex and age throughout adulthood. Clin Biomech (Bristol, Avon) 2015;30:22-7.

18. Garatachea N, Lucia A. Genes, physical fitness and ageing. Ageing Res Rev 2013;12:90-102.

19. Kalyani RR, Corriere M, Ferrucci L. Age-related and disease-related muscle loss: the effect of diabetes,

Cite this article as: Zhou W, Ma X, Pan L, Wang Y, Zhou C. Application of conventional ultrasound coupled with virtual touch tissue imaging and quantification in the assessment of muscle strength. Ann Palliat Med 2020;9(5):3402-3409. doi: 10.21037/ apm-20-1715 obesity, and other diseases. Lancet Diabetes Endocrinol 2014;2:819-29.

20. Zhao Q, Yang ST, Wang JJ, et al. TNF alpha inhibits myogenic differentiation of $\mathrm{C} 2 \mathrm{C} 12$ cells through $\mathrm{NF}-\kappa \mathrm{B}$ activation and impairment of IGF-1 signaling pathway. Biochem Biophys Res Commun 2015;458:790-5.

21. Brandenburg JE, Eby SF, Song P, et al. Feasibility and reliability of quantifying passive muscle stiffness in young children by using shear wave ultrasound elastography. J Ultrasound Med 2015;34:663-70.

22. Du LJ, He W, Cheng LG, et al. Ultrasound shear wave elastography in assessment of muscle stiffness in patients with Parkinson's disease: a primary observation. Clin Imaging 2016;40:1075-80. 